INSTITUT NATIONAL DE RECHERCHE EN INFORMATIQUE ET EN AUTOMATIQUE

\title{
Optimal control of a parabolic equation with time-dependent state constraints
}

J. Frédéric Bonnans — Pascal Jaisson

\section{$\mathbf{N}^{\circ} 6784$}

Décembre 2008

Thème NUM

\section{apport}

derecherche 



\title{
Optimal control of a parabolic equation with time-dependent state constraints
}

\author{
J. Frédéric Bonnans *, Pascal Jaisson \\ Thème NUM — Systèmes numériques \\ Équipes-Projets Commands \\ Rapport de recherche $n^{\circ} 6784$ - Décembre 2008 - 24 pages
}

\begin{abstract}
In this paper we study the optimal control problem of the heat equation by a distributed control over a subset of the domain, in the presence of a state constraint. The latter is integral over the space and has to be satisfied at each time. Using for the first time the technique of alternative optimality systems in the context of optimal control of partial differential equations, we show that both the control and multiplier are continuous in time. Under some natural geometric hypotheses, we can prove that extended polyhedricity holds, allowing to obtain no-gap second-order optimality conditions, that characterize quadratic growth. An expansion of the value function and of approximate solutions can be computed for a directional perturbation of the r.h.s. of the state equation.
\end{abstract}

Key-words: Optimal control, parabolic equations, state constraints, alternative optimality system, quadratic growth, second-order optimality conditions, sensitivity, expansion of value function and solutions.

* INRIA-Saclay and Centre de Mathématiques Appliquées, Ecole Polytechnique, route de Saclay, 91128 Palaiseau, France. Email: Frederic.Bonnans@inria.fr.

$\dagger$ Laboratoire de Mathématiques Appliquées, Université de Versailles Saint-Quentin, 45 avenue des Etats Unis 78035 Versailles, France. Email: pascal.jaisson@math.uvsq.fr.

Centre de recherche INRIA Saclay - Île-de-France

Parc Orsay Université

4, rue Jacques Monod, 91893 ORSAY Cedex

Téléphone : +33172925900 


\section{Commande optimale d'une équation parabolique avec contraintes sur l'état dépendant du temps}

Résumé : Cet article étudie le problème de la commande optimale de l'équation de la chaleur par un contrôle distribué sur une partie du domaine, en présence d'une contrainte sur l'état. Cette dernière est intégrale en espace et doit être respectée à chaque instant. Utilisant pour la première fois la technique des multiplicateurs alternatifs dans le contexte de la commande optimale des équations aux dérivées partielles, nous montrons la continuité en temps de la commande et du multiplicateur. Sous des hypothèses naturelles, la condition de polyédricité étendue est satisfaite, ce qui permet d'obtenir des conditions d'optimalité du second ordre sans écart, qui caractérisent la croissance quadratique. On en déduit également un développement de la valeur et des solutions approchées pour une perturbation directionnelle du second membre de l'équation d'état.

Mots-clés : Commande optimale, équations paraboliques, contraintes sur l'état, système d'optimalité alternatif, croissance quadratique, conditions d'optimalité du second ordre, sensibilité, développement de la valeur et de la solution. 


\section{Introduction}

Optimal control problems of PDE (partial differential equations) with state constraints have been intensively studied since the eighties, starting with the work by Bonnans and Casas [8, 11, 12] and then Abergel and Temam [1]. More specifically, problems with mixed control and state constraints, or with "pure" state constraints, for parabolic systems have been studied in Casas 20, where a regularity result for the costate equation with r.h.s. measure was obtained, and Casas, Raymond and Zidani [32, 19]. The Stone-Čech compactification theorem has been introduced for deriving optimality conditionsfor parabolic problems with pure or mixed state constraints by Arada and Raymond 6, 5, 3, 4, We note the recent study by Casas, De los Reyes and Tröltzsch 21] on sufficient conditions.

In this paper the system is described by a nonlinear parabolic equation whose properties are recalled in section 2 We consider the case of a standard quadratic control and of a finite number of state constraints for each time; our model example is the one of a unique state constraint which is the $L^{2}$ norm (integration w.r.t. space only). This allows us in section 3 to fully characterize qualification and to use the alternative formulation of the optimality conditions, introduced in Jacobson, Lele and Speyer [25, rigorously obtained in Maurer [31] and generalized in Bonnans and Hermant [14] to the case of an arbitrary number of state constraints; see also Hager [24] in the case of first-order state constraints. Thanks to this alternative formulation, we are able to prove that both the multiplier associated with the state constraint and the optimal control are timecontinuous, and to obtain the expression of these two "algebraic variables" as fonctions of the state and alternative costate. Then, under some geometric hypotheses (finitely many junction points and growth condition for the state constraint), we obtain in section 4 no-gap second-order optimality conditions. Finally in section 5 we perform a sensitivity analysis w.r.t. the r.h.s. of the state equation. We obtain under weak assumptions the second-order expansion of the value function as well as the first-order expansion of the soltion path.

\section{Framework}

Consider the heat equation with distributed control over a subset of the domain:

$$
\begin{aligned}
y_{t}-\Delta y+\gamma y^{3} & =i_{\omega} u \text { in } Q, \\
y & =0 \text { over } \Sigma, \\
y(\cdot, 0) & =y_{0} \text { over } \Omega,
\end{aligned}
$$

where $\gamma \in \mathbb{R}, \Omega$ is an open set of $\mathbb{R}^{n}, n \in\{2,3\}$ with $C^{2}$-smooth boundary $\partial \Omega$, $Q:=\Omega \times[0, T], \Sigma:=\partial \Omega \times[0, T], \omega$ is an open subset of $\Omega, Q_{\omega}=\omega \times[0, T], u \in$ $L^{2}\left(Q_{\omega}\right)$ is the control and $T>0$ is the horizon. The function $i_{\omega}$ is the injection from $L^{2}\left(Q_{\omega}\right)$ into $L^{2}(Q)$ and the given initial state $y_{0}$ belongs to $H^{1}(\Omega)$. Let

$$
\left\{\begin{array}{l}
H^{2,1}(Q):=\left\{y \in L^{2}\left(0, T, H^{2}(\Omega)\right) ; y_{t} \in L^{2}(Q)\right\} \\
H_{\Sigma}^{2,1}(Q):=\left\{y \in H^{2,1}(Q) ; y=0 \text { over } \Sigma\right\}
\end{array}\right.
$$

Norms for functions depending on space variables, or depending only on time, will be denoted by simple bars, and the $L^{s}$ norms will be, if there is no ambiguity, denoted by subscript $s$. We recall the following inclusions (see Lions and 
Magenes 30] and Adams [2, resp.)

$$
\begin{gathered}
H^{2,1}(Q) \subset C\left([0, T], H_{0}^{1}(\Omega)\right) \text { with compact inclusion, } \\
H^{1}(\Omega) \subset L^{6}(\Omega) \text { and } \quad|z|_{6} \leq c_{2}|\nabla z|_{2}, \quad \text { for all } z \in H_{0}^{1}(\Omega) \text {, when } n \leq 3 .
\end{gathered}
$$

We say that $y \in H^{2,1}(Q)$ is a state associated with $u \in L^{2}(Q)$ if $(y, u)$ satisfies the state equation (1)-(3). The following is known (see 29] and for a similar result in the case of boundary control [10]).

Lemma 1. For any given $q \in L^{\infty}\left(0, T, L^{3}(\Omega)\right), f \in L^{2}(\Omega)$, and $f_{0} \in H_{0}^{1}(\Omega)$, the following equation has a unique solution $z$ in $H_{\Sigma}^{2,1}(Q)$ :

$$
\begin{aligned}
z_{t}-\Delta z+q z & =f \text { in } Q \\
y(\cdot, 0) & =f_{0} \text { over } \Omega
\end{aligned}
$$

Proof. Set $c_{1}:=|q|_{L^{\infty}\left(0, T, L^{3}\right)}$. Multiplying (7) by $z$, integrating over space, and adopting the convention to eliminate the space argument when no confusion can occur, get

$$
\begin{aligned}
\frac{1}{2} \frac{\mathrm{d}}{\mathrm{d} t}|z(t)|_{2}^{2}+|\nabla z(t)|^{2} & =\int_{\Omega}\left(f(x, t) z(x, t)-q(x, t) z^{2}(x, t)\right) \mathrm{d} x \mathrm{~d} t \\
& \leq\left(|f(t)|_{2}+c_{1}|z(t)|_{6}\right)|z(t)|_{2}
\end{aligned}
$$

By (6) and Hölder's inequality, for all $\varepsilon>0$, we have that

$$
2|z(t)|_{6}|z(t)|_{2} \leq \varepsilon|z(t)|_{6}^{2}+\frac{1}{\varepsilon}|z(t)|_{2}^{2} \leq \varepsilon c_{2}^{2}|\nabla z(t)|_{2}^{2}+\frac{1}{\varepsilon}|z(t)|_{2}^{2} .
$$

Taking $\varepsilon$ such that $\varepsilon c_{1} c_{2}^{2}=1$, obtain with (9)

$$
\frac{\mathrm{d}}{\mathrm{d} t}|z(t)|_{2}^{2}+|\nabla z(t)|^{2} \leq 2|f(t)|_{2}|z(t)|_{2}+\frac{c_{1}}{\varepsilon}|z(t)|_{2}^{2} \leq|f(t)|_{2}^{2}+\left(1+\frac{c_{1}}{\varepsilon}\right)|z(t)|_{2}^{2} .
$$

By Gronwall's lemma, we have that for some $c_{3}>0$ :

$$
\|z\|_{L^{\infty}\left(0, T, L^{2}(\Omega)\right.}^{2}+\|\nabla z\|_{2}^{2} \leq c_{3}\left(\left|f_{0}\right|_{2}^{2}+\|f\|_{L^{2}(Q)}^{2}\right) .
$$

In particular when $f=0$ and $f_{0}=0$, this proves the uniqueness property. Integrating (11) over time and taking (11) into account, we obtain an apriori estimate of $z$ in the space

$$
W(0, T):=\left\{z \in L^{\infty}\left(0, T, L^{2}(\Omega)\right) ; \quad \nabla z \in L^{2}\left(0, T, L^{2}(\Omega)\right)\right\},
$$

therefore also with (6) of $z$ in $L^{2}\left(0, T, L^{6}(\Omega)\right)$, and finally of $q z$ in $L^{2}(\Omega)$. By (71) we get an apriori estimate of $z$ in $H_{\Sigma}^{2,1}(Q)$. The construction of the solution is then easily obtained by a Galerkin approximation.

Lemma 2. For given $u \in L^{2}\left(Q_{\omega}\right)$, either the state equation has a unique solution, or there exists a maximal time $\tau \in(0, T]$ such that the state equation with time restricted to $[0, \tau-\varepsilon]$ has, for all $\varepsilon>0$, a unique solution, and $|y(t)|_{6}$ is not bounded over $[0, \tau)$. 
Proof. a) Existence of a solution of the state equation for small time. Let $a_{0}:=\left|y_{0}\right|_{6}$ and, for $b>a_{0}$, let $\varphi_{b}: \mathbb{R}_{+} \rightarrow \mathbb{R}_{+}$be defined by

$$
\varphi(s)= \begin{cases}1 & \text { when } s \leq b \\ \max (1+b-s, 0) & \text { when } s>b\end{cases}
$$

Consider the perturbed state equation

$$
\begin{aligned}
y_{t}-\Delta y+\gamma \varphi\left(|y|_{6}\right) y^{3} & =i_{\omega} u \text { in } Q \\
y(\cdot, 0) & =y_{0} \text { over } \Omega,
\end{aligned}
$$

with possible solution in $H_{\Sigma}^{2,1}(Q)$. Since $\varphi$ has value in $[0,1]$ and $\varphi(a)=0$ when $a \geq b+1$, we have that $\varphi\left(|y|_{6}\right) y^{3} \in L^{\infty}\left(0, T, L^{2}(\Omega)\right)$. It is therefore easy to obtain an a priori estimate of the solution in $H_{\Sigma}^{2,1}(Q)$ and to construct a solution by Galerkin's method; when passing to the limit in the nonlinear term, we use the compact inclusion (5) and the continuity of $\varphi$. Since $|y|_{6}$ is a continuous function of time, we have that $|y|_{6} \leq b$ when $t \leq t_{1}$, for some $t_{1}>0$. This proves that the state equation has a solution for $t \leq t_{1}$.

b) Continuation. Let $y$ be a solution of the state equation for time $t \leq t_{0}$. If the continuous function $|y(t)|_{6}$ is bounded, then $y$ has a limit $y\left(t_{0}\right)$. Applying the construction of step a with $b>\max _{0 \leq t \leq t_{0}}|y(t)|_{6}$, we can construct a solution for $t>t_{0}$, close to $t_{0}$. This proves that either the solution is defined over $[0, T]$, or there exists $\tau$ such that the solution is defined over $[0, \tau)$, and $\lim \sup _{t \uparrow \tau}|y(t)|_{6}=$ $+\infty$.

c) Uniqueness of the solution. If $y_{1}$ and $y_{2}$ are two solution over $\left(0, t_{0}\right)$, setting $z:=y_{2}-y_{1}$ and $q=\gamma\left(3 y^{2}+3 y z+z^{2}\right)$, we obtain that $q \in L^{\infty}\left(0, t_{0}, L^{3}(\Omega)\right)$. Applying lemma 1 with $f=0$ and $f_{0}=0$, and $T=t_{0}$, obtain $z=0$, which is the desired uniqueness property. In particular, let $y_{i}$ be solutions of the state equation for maximal time $t \leq t_{i}, i=1,2$. Then $y_{1}$ and $y_{2}$ coincide over $\left(0, \min \left(t_{1}, t_{2}\right)\right)$. If say $t_{1}<t_{2}$ if follows that $\left|y_{1}(t)\right|_{6}$ remains bounded for $t \leq t_{1}$ which is impossible. Hence there is a unique solution defined up to its maximal time.

In view of the estimate

$$
\frac{1}{2} \frac{\mathrm{d}}{\mathrm{d} t}\left|y_{u}(t)\right|^{2}+\left|\nabla y_{u}(t)\right|^{2}+\gamma\left|y_{u}(t)\right|_{4}^{4}=\int_{\omega} y_{u}(x, t) u(x, t) \mathrm{d} x \mathrm{~d} t,
$$

we have, when $\gamma \geq 0$, given $u \in L^{2}(Q)$, an apriori estimate of $y_{u}$ in $W(0, T)$; using a Galerkin approximation argument, one easily deduces that when $\gamma \geq 0$ the state equation has a unique solution $y_{u}$, and that there exists $c>0$ not depending on $\gamma$ such that $\|y\|_{H^{2,1}(Q)} \leq c\|u\|_{2}$. When $\gamma<0$, the state equation has at most one solution. The strong solution, always defined for small time, can explode in a finite time, see for similar results Bebernes and Kassoy 7 and Tartar [35. In any case we denote by $y_{u}$ the solution. The implicit function theorem can be applied to the state equation (see [9, 29]), and since the latter is of class $C^{\infty}$, we obtain that the mapping $u \rightarrow y_{u}$ is of class $C^{\infty}$.

The associated cost function is, for some $N>0$ :

$$
J(u, y)=\frac{1}{2} \int_{Q}\left(y(x, t)-y_{d}(x, t)\right)^{2} \mathrm{~d} x \mathrm{~d} t+\frac{N}{2} \int_{Q_{\omega}} u^{2}(x, t) \mathrm{d} x \mathrm{~d} t .
$$


For some $C>\frac{1}{2} \int_{\Omega}\left|y_{0}(x)\right|^{2} \mathrm{~d} x$, define $g: L^{2}(\Omega) \rightarrow \mathbb{R}$ by $g(z):=\frac{1}{2} \int_{\Omega}|z(x)|^{2} \mathrm{~d} x-$ $C$. Consider the state constraint

$$
g(y(\cdot, t)) \leq 0 .
$$

The paper is concerned with the discussion of optimality conditions of the following optimal control problem:

$$
\operatorname{Min}_{(u, y) \in L^{2}\left(Q_{\omega}\right) \times H^{2,1}(Q)} J(u, y) \text { subject to (1)-(3) and (19). }
$$

Remark 3. When $\gamma \geq 0$, problem $(P)$ is always feasible and has a nonempty set of solutions. Indeed, it follows from (17) that $t \mapsto g\left(y_{u}\right)(t)$ is nonincreasing when $\gamma \geq 0$ and $u=0$. In particular, $u=0$ is feasible when $\gamma \geq 0$. The existence of a solution to $(P)$ can be easily proved by passing to the limit in a minimizing sequence, since when $\gamma \geq 0$, by (17), a minimizing sequence of ocontrols is bounded in $L^{2}\left(Q_{\omega}\right)$ and hence the corresponding sequence of states is bounded in $y$ in $H^{2,1}(Q)$ When $\gamma<0$ the existence of a solution is unclear. Of course it makes sense to study optimality conditions even if we cannot prove the existence of a solution.

A trajectory of $(P)$ is an element $(u, y) \in L^{2}\left(Q_{\omega}\right) \times H^{2,1}(Q)$ satisfying the state equation (11)-(3). A feasible trajectory is one satisfying the state constraint (19). The set of feasible trajectories is denoted $F(P)$.

We say that $(\bar{u}, \bar{y})$ is a local solution of $(P)$ that satisfies the quadratic growth condition with parameter $\theta \in \mathbb{R}$, if it belongs to $F(P)$ and there exists $\rho>0$ such that

$$
J(\bar{u}, \bar{y}) \geq J(u, y)+\theta|\bar{u}-u|_{L^{2}\left(Q_{\omega}\right)}^{2} \text { if }(u, y) \in F(P) \text { and }|u-\bar{u}|_{L^{2}\left(Q_{\omega}\right)} \leq \rho .
$$

If this holds for $\theta=0$, we say that $(\bar{u}, \bar{y})$ is a local solution of $(P)$. We say that $(\bar{u}, \bar{y}) \in F(P)$ satisfies the quadratic growth condition if (20) holds for some $\theta>0$ and $\rho>0$. For $(u, y) \in F(P)$, define the contact set by

$$
I(g(y))=\{t \in[0, T] ; g(y)(t)=0\} .
$$

For $t \in[0, T]$, if $g(y)(t)=0$ (resp. $g(y)(t)<0$ ), we say that the constraint is active (resp. inactive) at time $t$. Finally we denote by $\chi_{\omega}$ the restriction $L^{2}(\Omega) \rightarrow L^{2}(\omega)$ (which is nothing else than the transposition of $i_{\omega}$ ).

\section{$3 \quad$ First-order analysis}

\subsection{The unqualified optimality system}

We first put problem $(P)$ under a compact format by defining

$$
\left\{\begin{array}{l}
G: L^{2}\left(Q_{\omega}\right) \times\left\{y \in H^{2,1}(Q) \text { s.t. } y_{\mid \Sigma}=0\right\} \rightarrow L^{2}(Q) \times H_{0}^{1}(\Omega), \\
G(u, y):=\left(\begin{array}{c}
y_{t}-\Delta y+\gamma y^{3}-i_{\omega} u \\
y(\cdot, 0)-y_{0}
\end{array}\right) .
\end{array}\right.
$$

The linearized state equation at point $(u, y)$ is defined as

$$
z_{t}-\Delta z+3 \gamma y_{u}^{2}=i_{\omega} v \text { in } Q ; \quad z=0 \text { on } \Sigma, \quad z(\cdot, 0)=0 .
$$


This linearized state equation is well-posed in the sense that, with each $v \in$ $L^{2}\left(Q_{\omega}\right)$ is associated a unique solution $z \in H^{2,1}(Q)$. Since $G$ is a $C^{\infty}$ mapping, it follows from the Implicit Function Theorem that the mapping $u \rightarrow y_{u}$ is of class $C^{\infty}: L^{2}\left(Q_{\omega}\right) \rightarrow H^{2,1}(Q)$, and the directional derivative of the state at point $u$ in direction $v$ is the solution $z$ of the linearized state equation. Next define $\mathcal{J}: L^{2}\left(Q_{\omega}\right) \rightarrow \mathbb{R}$ and $\mathcal{G}: L^{2}\left(Q_{\omega}\right) \rightarrow C([0, T])$ by

$$
\mathcal{J}(u):=J\left(u, y_{u}\right) ; \quad \mathcal{G}(u)(t):=g\left(y_{u}(t)\right)=\frac{1}{2}\left|y_{u}(t)\right|^{2}-C .
$$

We can rewrite problem $(P)$ under the form of an "abstract problem":

$$
\operatorname{Min}_{u} \mathcal{J}(u) ; \quad \mathcal{G}(u) \in K
$$

where $K=C([0, T])_{-}$is the cone of continuous nonpositive functions over $[0, T]$. We recall that the topological dual of $C([0, T])$ is the set $M(0, T)$ of regular Borel measures over $[0,1]$. Therefore the (negative) polar cone of $K$ is $K^{-}=M([0, T])_{+}$. We know (see e.g. [18 Example 2.62 and 2.63]) that since $K$ is a cone, its normal cone at a point $h \in K$ is $N_{K}(h)=K^{-} \cap h^{\perp}$ and that for $h \in K$, denoting by $\operatorname{supp}(\mu)$ the support of a measure $\mu$ :

$$
N_{K}(h)=\left\{\mu \in M(0, T)_{+} ; \operatorname{supp}(\mu) \subset h^{-1}(0)\right\} .
$$

Define (see e.g. [18, Section 3.1.2]) the generalized Lagrangian of problem $(A P)$ as $\mathcal{L}: L^{2}\left(Q_{\omega}\right) \times \mathbb{R} \times M([0, T])$ such that

$$
\mathcal{L}(u, \alpha, \mu):=\alpha \mathcal{J}(u)+\langle\mu, \mathcal{G}(u)\rangle
$$

and the set of generalized Lagrange multipliers associated with $u \in F(A P)$ as

$$
\Lambda_{g}(u):=\left\{(\alpha, \mu) \in \mathbb{R}_{+} \times N_{K}(\mathcal{G}(u)) ;(\alpha, \mu) \neq 0 ; D_{u} \mathcal{L}(u, \alpha, \mu)=0\right\} .
$$

This set is a (possibly empty) convex cone.

Theorem 4. With a local solution $(u, y)$ of $(P)$ is associated a non empty set of generalized Lagrange multipliers.

Proof. This results from [18. Prop. 3.18]. For applying this result it suffices to verify that the cone defined in equation (3.24) of 18 has a nonempty relative interior, which trivially holds since $K$ has a nonempty interior.

We next give a more explicit form to the optimality conditions. The condition $\mu \in N_{K}(\mathcal{G}(u)$ is equivalent in view of (25) to

$$
g(y) \leq 0, \quad \mu \geq 0, \quad \int_{0}^{T} g(y(t)) \mathrm{d} \mu(t)=0 .
$$

By (26), $D_{u} \mathcal{L}(u, \alpha, \mu)=0$ iff, for $y=y_{u}$ :

$$
\alpha N \int_{Q_{\omega}} u v+\alpha \int_{Q}\left(y-y_{d}\right) z_{v}+\int_{0}^{T} \int_{\Omega} y z_{v} \mathrm{~d} \mu=0, \quad \text { for all } v \in L^{2}\left(Q_{\omega}\right),
$$

where $z_{v}$ is the solution of the linearized state equation (23). For the sake of simplicity we will assume in the sequel of this paper that the state constraint is 
not active at the final time. Define the costate $p$ as the solution in $L^{2}(Q)$ of the linear equations

$$
\left\{\begin{array}{l}
\int_{Q}\left[\alpha\left(y-y_{d}\right) z+p\left(\Delta z-3 \gamma y^{2} z-z_{t}\right)\right] \mathrm{d} x \mathrm{~d} t+\int_{Q} y(x, t) z(x, t) \mathrm{d} x \mathrm{~d} \mu(t)=0 \\
\text { for all } z \in Z:=\left\{H_{\Sigma}^{2,1}(Q) ; z(\cdot, 0)=0\right\} .
\end{array}\right.
$$

Setting $f=z_{t}-\Delta z+3 \gamma y^{2} z$, this is equivalent to

$$
\int_{Q} p f=\alpha \int_{Q}\left(y-y_{d}\right) z+\int_{Q} y(x, t) z(x, t) \mathrm{d} x \mathrm{~d} \mu(t) .
$$

For given $f \in L^{2}(Q)$, in view of lemma 1 there exists a unique $z \in Z$ such that $z_{t}-\Delta z+3 \gamma y^{2} z=f$. Therefore the r.h.s. of (31) may be interpreted as a continuous linear form over $L^{2}(Q)$. By the Riesz theorem there exists a unique $p \in L^{2}(Q)$ such that (31) holds for all $f \in L^{2}(Q)$, or equivalently, (30) has a unique solution $p \in L^{2}(Q)$. Let $\mathcal{D}(Q)$ denote the set of $C^{\infty}$ functions in $Q$ with compact support, and let $\mathcal{D}^{\prime}(Q)$ be the associated dual set of distributions. We see that (30) is (formally for the two last equations) equivalent to

$$
\begin{aligned}
-p_{t}-\Delta p+3 \gamma y^{2} p & =\alpha\left(y-y_{d}\right)+y \mathrm{~d} \mu(t) \text { in } \mathcal{D}^{\prime}(Q), \\
p(\cdot, T) & =0 \\
p & =0 \text { on } \Sigma .
\end{aligned}
$$

We can justify (33) and (34) by observing that, since $p$ is solution of a backwards heat equation with r.h.s. measure, by [20, it belongs to the space $L^{r}\left(0, T, W^{1, q}(\Omega)\right)$, for all $r, q$ in $[1,2)$ such that $2 / r+n / p>n+1$, and hence, its traces at time $T$ and on $\Sigma$ are well-defined. Actually we will derive in a direct manner a stronger regularity result in section 3.3

When $f=i_{\omega} v$, for some $v \in L^{2}\left(Q_{\omega}\right)$, substracting (30) from (29), and taking (23) into account, obtain $\int_{Q_{\omega}}(\alpha N u v+p v)=0$, for all $v \in L^{2}\left(Q_{\omega}\right)$, or equivalently

$$
\alpha N u+\chi_{\omega} p=0 \quad \text { a.e. over } Q_{\omega} .
$$

Finally we have proved that

$$
\Lambda_{g}(u):=\left\{\begin{array}{l}
(\alpha, \mu) \in \mathbb{R}_{+} \times N_{K}(\mathcal{G}(u)) ; \quad(\alpha, \mu) \neq 0 \\
\chi_{\omega} p=-\alpha N u, \text { where } p \text { is solution of }
\end{array}\right\} .
$$

Denote the set of singular multipliers and Lagrange multipliers, resp., by

$$
\begin{aligned}
& \Lambda_{s}(u):=\left\{\mu \in N_{K}(\mathcal{G}(u)) ; \mu \neq 0 ; D_{u} \mathcal{L}(u, 0, \mu)=0\right\}, \\
& \Lambda_{\ell}(u):=\left\{\mu \in N_{K}(\mathcal{G}(u)) ; D_{u} \mathcal{L}(u, 1, \mu)=0\right\} .
\end{aligned}
$$

A nonsingular multiplier is of the form $\alpha\left(1, \mu^{\prime}\right)$ where $\mu^{\prime}:=\mu / \alpha$ is a Lagrange multiplier. Denote by $\operatorname{supp}(\mu) \subset[0, T]$ the support of the measure $\mu$, and let the singular contact set be defined by

$$
I_{s}(u)=\left\{t \in I\left(g\left(y_{u}\right)\right) ; y_{u}(\cdot, t)=0 \text { a.e. on } \omega\right\} .
$$

In view of (17), we see that the singular contact set is the set of times for which the constraint is active and the control has no influence on its time derivative. We next characterize the absence of singular mulitpliers using the following concept. We will say that the state constraint is nondegenerate at point $u$ if $I_{s}(u)=\emptyset$; otherwise we say that the state constraint is degenerate. 
Theorem 5. Let $(u, y)$ be a feasible point of $(P)$. Then $\Lambda_{s}(u)$ is empty iff the state constraint is nondegenerate at point $u$.

Proof. Let $\mu \in \Lambda_{s}(u)$. By (35), $p=0$ over $Q_{\omega}$. Then by (32), $y d \mu=0$ over $Q_{\omega}$, which means that $\operatorname{supp}(\mu) \subset I_{s}(u)$. Since $\mu \neq 0$, the state constraint is degenerate.

Conversely, if the state constraint is degenerate, there exists $\tau \in I_{s}(u)$. Let $\mu$ be the Dirac measure at time $\tau$. Then the costate equation is satisfied, as well as (35), by $p=0$. Therefore $\mu$ is a singular multiplier.

\subsection{Constraint qualification condition}

By a constraint qualification condition one usually understands a condition implying that Lagrange multipliers associated to a local solution exist. Combining theorems 4 and 5 we obtain that nondegeneracy of the constraint is a qualification condition. Yet it is of interest to relate it to the standard Robinson constraint qualification (see $[33,[34]$ ). Since $C([0, T])$ - has a non empty interior, the latter writes for a given $(u, y) \in F(P)$ :

$\left\{\right.$ There exists $(v, z) \in L^{2}\left(Q_{\omega}\right) \times H^{2,1}(Q)$, solution of (23),

\{ such that $g^{\prime}(y(t)) z(t)=\int_{\omega} y(x, t) z(x, t) \mathrm{d} x<0$, for all $t \in I(g(y))$.

Remark 6. (i) It is know that the constraint qualification implies that the set of Lagrange multipliers associated with a local solution is non empty and bounded. (ii) In addition, in the case of a constraint to belong to a convex set with a nonempty interior, then by [36] (see also [18, Prop. 3.17]), constraint qualification holds at a local solution iff the set of Lagrange multipliers is non empty and bounded.

Theorem 7. Let $(u, y) \in F(P)$. Then the state constraint is not degenerate iff the constraint qualification condition (40) holds.

The proof is based on the following lemma, where we show how to control the time derivative of the linearized state constraint. We first need to set (here $C$ is the constant used in the definition of the state constraint):

$$
\begin{array}{r}
\mathcal{T}(y):=\left\{t \in[0, T] ; \quad \int_{\omega} y^{2}(x, t) d x \geq C,\right. \\
\kappa(t):= \begin{cases}\left(\int_{\omega} y^{2}(x, t) d x\right)^{-1} & \text { if } t \in \mathcal{T}(y), \\
0 & \text { otherwise, }\end{cases}
\end{array}
$$

and for $z \in H^{2,1}(Q)$

$$
\begin{aligned}
\eta(z, t):=-\kappa(t) \quad\left(\int_{\omega} z(x, t) u(x, t) \mathrm{d}\right. & t-2 \int_{\Omega} \nabla y(x, t) \nabla z(x, t) \mathrm{d} t \\
& \left.+4 \gamma \int_{\Omega} y^{3}(x, t) z(x, t) \mathrm{d} t\right) .
\end{aligned}
$$

Lemma 8. Let $(u, y) \in L^{2}(Q) \times H^{2,1}(Q)$. Then (i) For all $f \in L^{2}(Q)$, the problem

$$
\begin{array}{r}
z_{t}-\Delta z+3 \gamma y^{2} z=\eta(z, t) \chi_{\omega} y(x, t)+f(x, t) \\
z\left(x, t_{1}\right)=z^{0}, \forall x \in \Omega,
\end{array}
$$


has a unique solution in $H_{\Sigma}^{2,1}(Q)$, the mapping $f \mapsto z$ is continuous, and

$$
\frac{\mathrm{d}}{\mathrm{d} t} g^{\prime}(y) z=\int_{\Omega} f y \quad \text { over } \mathcal{T}(y)
$$

(ii) In particular, given $\nu \in L^{2}(0, T)$, when $f=\nu(t) \kappa(t) \chi_{\omega} y(x, t)$, we have that $f \in L^{2}(Q)$ and

$$
\frac{\mathrm{d}}{\mathrm{d} t} g^{\prime}(y) z=\nu(t) \quad \text { over } \mathcal{T}(y)
$$

Proof. (i) For $a \in H^{1}(0, T)$, nonnegative and nondecreasing, and $z$ in $H^{2,1}(Q)$, set $w=e^{-a(t)} z$. Then $w \in H^{2,1}(Q)$, and since $z_{t}=e^{a(t)}\left(w_{t}+\dot{a} w\right), z$ is solution of (45) iff $w$ is solution of

$$
\begin{aligned}
w_{t}+\dot{a} w-\Delta w+3 \gamma y^{2} w & =\eta(w, t) \chi_{\omega} y+e^{-a(t)} f \\
w(x, 0) & =e^{-a(t)} z_{0}, \forall x \in \Omega .
\end{aligned}
$$

Multiplying both sides of (48) by $w$ and integrating over space, obtain (skipping arguments of functions)

$$
\begin{aligned}
& \frac{1}{2} \frac{d}{d t}|w|_{2}^{2}+\int_{\Omega}\left(\dot{a}(t) w^{2}+|\nabla w|^{2}+3 \gamma y^{2} w^{2}\right)= \\
& \quad+\kappa(t)\left(\int_{\omega} w u-2 \int_{\Omega} \nabla y \nabla w+2 \gamma \int_{\Omega} y^{3} w\right) \int_{\omega} y w+e^{-a(t)} \int_{\Omega} f w .
\end{aligned}
$$

Using $a \geq 0, y \in H^{2,1}(Q) \subset C\left([0, T], H^{1}(\Omega)\right) \subset C\left([0, T], L^{6}(\Omega)\right), \kappa(t) \leq 2 C$, and the Cauchy-Schwartz inequality, obtain that for some $c_{1}>0$ :

$$
\begin{aligned}
\frac{1}{2} \frac{d}{d t}|w|_{2}^{2}+\dot{a}(t)|w|_{2}^{2}+|\nabla w|_{2}^{2} \leq & c_{1}\left(|w|_{2}^{2}|u|_{2}+\alpha|\nabla w|_{2}^{2}+\frac{1}{\alpha}|w|_{2}^{2}+|w|_{2}^{2}\right) \\
& +\frac{1}{2}\left(|f|_{2}^{2}+|w|_{2}^{2}\right) .
\end{aligned}
$$

Setting $\alpha:=\frac{1}{2 c_{1}}$, obtain, for some $c_{2}>0$ :

$$
\frac{d}{d t}|w|_{2}^{2}+\frac{1}{2}|\nabla w|_{2}^{2} \leq|w|_{2}^{2}\left(c_{1}|u|_{L^{2}(\omega)}+c_{2}-\dot{a}(t)\right)+|f|_{2}^{2}
$$

Choosing $a(t)=c_{1} \int_{t_{1}}^{t}|u|_{2}+c_{2}\left(t-t_{1}\right)$, for all $t \in(0, T)$, (this is indeed in $H^{1}(0, T)$, nonnegative and nondecreasing), obtain after a time integration

$$
\left|w\left(t_{2}\right)\right|_{2}^{2}+\left\|\nabla w\left(t_{2}\right)\right\|_{L^{2}\left(\Omega \times\left(t_{1}, t_{2}\right)\right)}^{2} \leq\left|z^{0}\right|_{2}^{2}+\|f\|_{L^{2}\left(\Omega \times\left(t_{1}, t_{2}\right)\right)}^{2} .
$$

So we have an a-priori estimate of $w$ in $L^{2}\left([0, T], H_{0}^{1}(\Omega)\right) \bigcap L^{\infty}\left([0, T], L^{2}(\Omega)\right)$. Let us show that (48) gives an a-priori estimate of $w_{t}-\Delta w$ in $L^{2}(Q)$. The a-priori estimate of $w$, and hence, of $z$, in $H^{2,1}(Q)$ will follow as well as the conclusion of the lemma. Indeed, since

$$
\left\|\eta(w, t) \chi_{\omega} y\right\|_{L^{2}(Q)}^{2} \leq\|y\|_{L^{\infty}\left(0, T, L^{2}(\Omega)\right)}^{2} \int_{0}^{T} \eta(w, t)^{2} \mathrm{~d} t,
$$

it suffices to prove that $\eta \in L^{2}(0, T)$. And this follows from

$$
\begin{aligned}
\eta(w, t)^{2} \leq C & \left(\|w\|_{L^{\infty}\left(0, T, L^{2}(\Omega)\right)}^{2} \mid u(t) \|_{2}^{2}+\right. \\
& \left.\|\nabla y\|_{L^{\infty}\left(0, T, L^{2}(\Omega)\right)}^{2}|\nabla w|_{2}^{2}+\|y\|_{L^{\infty}\left(0, T, L^{6}(\Omega)\right)}^{6}|w|_{2}^{2} \cdot\right) .
\end{aligned}
$$


Finally (46) follows from the fact that $\frac{\mathrm{d}}{\mathrm{d} t} g^{\prime}(y) z=\int_{\Omega}\left(y_{t} z+y z_{t}\right)$, using the expressions of $y_{t}$ and $z_{t}$, and (ii) is an easy consequence of (i).

Proof of theorem [7. If the state constraint is degenerate, the constraint qualification (40) obviously cannot hold. Assume now that the state constraint is nondegenerate. Set

$$
I_{\varepsilon}:=\{t \in[0, T] ; \operatorname{dist}(t, I(g(y))) \leq \varepsilon\} ; \quad J_{\varepsilon}:=[0, T] \backslash I_{\varepsilon} .
$$

Since $g(y(t))$ is continuous over $[0, T]$, we may take $\varepsilon>0$ so small that $I_{\varepsilon} \subset \mathcal{T}$, The set $J_{\varepsilon}$ is a finite union of relatively open subsets. Therefore $I_{\varepsilon}$ is the union finitely many closed intervals of the form $\left[a_{i}-\varepsilon, b_{i}+\varepsilon\right] \cap[0, T], i=1, \ldots, N$, and we have that $I(g(y)) \subset \cup_{i=1}^{N}\left[a_{i}, b_{i}\right]$. We next construct the perturbation $v$ as follows. Take $v=0$ over $J_{\varepsilon}$. Next, given $v$ and $z$ over $\left[0,\left(a_{i}-\varepsilon\right)_{+}\right]$, fix $v$ over $\left[a_{i}-\varepsilon, b_{i}+\varepsilon\right]$ such that, setting $\xi(t):=g^{\prime}(y(t)) z(t)$ is affine over $\left[a_{i}-\varepsilon, a_{i}\right]$ and has value -1 over $\left[a_{i}, b_{i}+\varepsilon\right]$. In view of lemma 8(ii), it suffices to take $z$ solution of (44)-(45), with $f=\nu(t) \kappa(t) \chi_{\omega} y(x, t)$, and with

$$
\nu(t):=\left\{\begin{array}{cl}
-\left(1+\xi\left(a_{i}-\varepsilon\right)\right) / \varepsilon, & t \in\left(a_{i}-\varepsilon, a_{i}\right), \\
0, & t \in\left(a_{i}, b_{i}+\varepsilon\right) .
\end{array}\right.
$$

The related control is $v=\eta(z, t) \chi_{\omega} y+f$.

In the sequel, we will say that $(u, y)$ is a qualified feasible point of $(P)$ if it satisfies (40), and a regular extremal if in addition it has an associated Lagrange multiplier.

\subsection{Alternative formulation of optimality conditions}

In this section we will assume that the state constraint is nondegenerate.

We recall the following integration by parts formula, see [23, Vol. I, p. 154]:

Lemma 9. Let $a$ and $b$ be two functions of bounded variations in $[0, T]$. Suppose that one is continuous, and the other is right-continuous. Then

$$
a\left(T^{-}\right) b\left(T^{-}\right)-a\left(0^{+}\right) b\left(0^{+}\right)=\int_{0}^{T} a(t) \mathrm{d} b(t)+\int_{0}^{T} b(t) \mathrm{d} a(t) .
$$

We apply this lemma to the expression $\int_{Q} y(x, t) z(x, t) \mathrm{d} x \mathrm{~d} \mu(t)$ appearing in the costate equation (30). The function $a(t):=\int_{\Omega} y(x, t) z(x, t) \mathrm{d} x$, with $z \in H^{2,1}(Q)$, is absolutely continuous; its derivative is

$$
\dot{a}(t)=\int_{\Omega}\left[y_{t}(x, t) z(x, t)+y(x, t) z_{t}(x, t)\right] \mathrm{d} x .
$$

Since $z(0)=0$ and taking the convention that $\mu(T)=0$, we see that the l.h.s. in (58) is zero, and hence,

$$
\left.\int_{0}^{T} \int_{\Omega} y(x, t) z(x, t) \mathrm{d} x \mathrm{~d} \mu(t)=-\int_{0}^{T} \int_{\Omega}\left[y_{t}(x, t) z(x, t)\right)+y(x, t) z_{t}(x, t)\right] \mu(t) \mathrm{d} x \mathrm{~d} t
$$


We now introduce the (first) alternative costate defined as the sum of the costate and of the product of the measure by the derivative (w.r.t. the state) of the state constraint; in other words

$$
p^{1}:=p+g^{\prime}(y) \mu=p+y \mu \text { in } L^{2}(Q) .
$$

Substracting (60) from (30), obtain

$$
\begin{aligned}
& \int_{Q} p^{1} z_{t} \mathrm{~d} x \mathrm{~d} t=\int_{Q}\left[\left(y-y_{d}\right)-3 \gamma y^{2} p-\mu y_{t}\right] z \mathrm{~d} x \mathrm{~d} t+\int_{Q} p \Delta z \mathrm{~d} x \mathrm{~d} t \\
& \text { for all } z \in Z:=\left\{z \in H^{2,1}(Q) ; z(\cdot, 0)=0 ; \quad z(x, t)=0 \text { on } \Sigma\right\} .
\end{aligned}
$$

Eliminating $p=p^{1}-y \mu$ and using $\int_{Q} y \Delta z \mathrm{~d} x \mathrm{~d} t=\int_{Q} z \Delta y \mathrm{~d} x \mathrm{~d} t$, we get

$$
\begin{aligned}
& \int_{Q} p^{1}\left(z_{t}-\Delta z+3 \gamma y^{2} z\right) \mathrm{d} x \mathrm{~d} t=\int_{Q}\left[\left(y-y_{d}\right)+\mu\left(3 \gamma y^{3}-\Delta y-y_{t}\right)\right] z \mathrm{~d} x \mathrm{~d} t \\
& \text { for all } z \in Z:=\left\{z \in H^{2,1}(Q) ; z(\cdot, 0)=0 ; \quad z(x, t)=0 \text { on } \Sigma\right\} .
\end{aligned}
$$

This is a classical adjoint equation with $L^{2}$ r.h.s.; using the expression of $y_{t}$, obtain the following result.

Lemma 10. Let $(u, y)$ be a regular extremal of $(P)$. The alternative multiplier $p^{1}=p+y \mu$ is the unique solution in $H^{2,1}(Q)$ of the equation

$$
\begin{aligned}
-p_{t}^{1}-\Delta p^{1}+3 \gamma y^{2} p^{1} & =y-y_{d}-\left(2 \Delta y-6 \gamma y^{3}+i_{\omega} u\right) \mu \quad \text { in } Q, \\
p^{1}(\cdot, T) & =0 \\
p^{1}(\cdot, t) & =0 \quad \text { on } \Sigma .
\end{aligned}
$$

Then, we have the following proposition stating the alternative formulation of the optimality conditions:

Proposition 11. Let $(u, y) \in F(P)$ be qualified. Then with $(u, y)$ is associated a Lagrange multiplier iff there exists a pair $\left(p^{1}, \mu\right) \in H^{2,1}(Q) \times M_{+}([0, T])$, satisfying the state equation (11)-(3), the alternative costate equation (64)-(66), the complementarity conditions (28) for the state constraint, and the relation

$$
N u+\chi_{\omega}\left(p^{1}-\mu y\right)=0 \text { a.e. on } \omega \times[0, T] .
$$

Proof. The necessity of the alternative costate equation (64)-(66) has been obtained in the above discussion. Eliminating $p=p^{1}-y \mu$ from (35), we obtain (67). Conversely, substituting $p^{1}=p+y \mu$ in the alternative optimality system, and using (60), we recover the "classical" adjoint equation (32)-(34) as well as (35).

We now prove the following regularity result.

Lemma 12. Let $(u, y)$ be a regular extremal of $(P)$ and $\left(p, p^{1}, \mu\right)$ the classical and alternative costate and the multiplier associated with the state constraint. Then (i) $\mu$ is a continuous function of time and $u \in C\left([0, T] ; H^{1}(\omega)\right)$, (ii) if at 
time $t$ the state constraint is active and $\int_{\omega} y^{2}(x, t) d x \neq 0$, then

$$
\begin{array}{r}
0=\frac{d}{d t}(g(y))(t)=-|\nabla y(t)|^{2}+\int_{\omega} u(x, t) y(x, t) \mathrm{d} x-\gamma|y(t)|_{4}^{4}, \\
\mu(t)=\frac{N|\nabla y(t)|^{2}+\gamma N|y(t)|_{4}^{4}+\int_{\omega} y(x, t) p^{1}(x, t) \mathrm{d} x}{\left|\chi_{\omega} y(t)\right|_{2}^{2}} \\
u=\frac{1}{N} \chi_{\omega}\left(\frac{N|\nabla y(t)|^{2}+\gamma N|y(t)|_{4}^{4}+\int_{\omega} y(x, t) p^{1}(x, t) \mathrm{d} x}{\left|\chi_{\omega} y(t)\right|_{2}^{2}} y-p^{1}\right) .
\end{array}
$$

Proof of lemma 12. (i) By (67), and since both $y$ and $p^{1}$ belong to $H^{2,1}(Q) \subset$ $C\left([0, T] ; H^{1}(\Omega)\right)$, it suffices to prove that $\mu$ is continuous to obtain the conclusion. Since $\mu$ has left and right limits, and $p^{1}$ as well as $y$ belong to $C\left(0, T, H_{0}^{1}(\Omega)\right)$, the same holds for $u$ by 67 ; the latter implies also, denoting by [.] the jump function (e.g., $\left.[u](t):=u\left(t^{+}\right)-u\left(t^{-}\right)\right)$, that $N[u]=[\mu] \chi_{\omega} y$. Taking the scalar product of both sides by $[u]$, get

$$
N|[u]|_{L^{2}(\omega)}^{2}=[\mu] \int_{\omega}[u] y \mathrm{~d} x .
$$

If $[\mu](t) \neq 0$ for some time $t$, then the constraint is active and attains its maximum at time $t$. The formal expression of the derivative of $g(y)$ is

$$
\frac{d}{d t}(g(y))(t)=\int_{\Omega} y \Delta y+\int_{\omega} u y-\gamma \int_{\Omega} y^{4}=-\int_{\Omega}|\nabla y|^{2}+\int_{\omega} u y-\gamma \int_{\Omega} y^{4}
$$

Since $y \in C\left(0, T, H^{1}(\Omega)\right)$ the first and the last terms in the r.h.s. are continuous (we recall that $H^{1}(\Omega) \subset L^{6}(\Omega)$ with continuous embedding in dimension 2 or $3)$; since $u$ has left and right limits, so has $\frac{\mathrm{d}}{\mathrm{d} t} g(y)$ and they are given by (72), where $u$ is the left or right limit, so that the jump is

$$
\left[\frac{d}{d t} g(y)(t)\right]=\int_{\omega}[u] y=\frac{N}{[\mu]}|[u]|_{L^{2}(\omega)}^{2} .
$$

Since $g(y)$ attains a maximum, $\left[\frac{d}{d t}(g(y))(t)\right] \leq 0$, and hence, $[u]=0$, contradicting $[\mu] \neq 0$. We have proved point (i) as well as (68).

(ii) Elimining the control variable from (67) and using (68), get (69). Eliminating then $\mu$ thanks to (69) in (67), obtain (70).

Lemma 13. Let $(u, y)$ be a regular extremal of $(P)$. Then $\left(p, p^{1}, \mu\right)$ the classical and alternative costate and the multiplier associated with the state constraint are unique.

Proof. Let $\left(p_{1}, p_{1}^{1}, \mu_{1}\right)$ and $\left(p_{2}, p_{2}^{1}, \mu_{2}\right)$ two triples of classical and alternative costates and multipliers associated with the state constraint. Set

$$
\left(\tilde{p}, \tilde{p}^{1}, \tilde{\mu}\right)=\left(p_{1}-p_{2}, p_{1}^{1}-p_{2}^{1}, \mu_{1}-\mu_{2}\right) .
$$

Then $\tilde{p}^{1}$ is solution of:

$$
\begin{aligned}
-\tilde{p}_{t}^{1}-\Delta \tilde{p}^{1}+3 \gamma y^{2} \tilde{p}^{1} & =-\left(2 \Delta y-6 \gamma y^{3}+i_{\omega} u\right) \tilde{\mu} \text { in } Q \\
\tilde{p}(\cdot, T) & =0 \\
\tilde{p}(\cdot, t) & =0 \text { on } \Sigma .
\end{aligned}
$$


So if $\tilde{\mu}=0$ it follows that $\tilde{p}^{1}=0$ and these two relations imply $\tilde{p}=0$. Therefore it suffices to prove that $\tilde{\mu}=0$. In view of the abstract form of the optimality system, we have that $\left\langle\tilde{\mu}, \mathcal{G}^{\prime}(u) v\right\rangle=0$ for all $v \in L^{2}\left(Q_{\omega}\right)$. Take $\varepsilon>0$ such that the state constraint is not active for time $t \leq \varepsilon$. The support of $\tilde{\mu}$ is concentrated over $[\varepsilon, T]$. By lemma 8 we can make $\mathcal{G}^{\prime}(u) v$ equal to an arbitrary function of $H^{1}(\varepsilon, T)$ ), and since the inclusion of $H^{1}(\varepsilon, T)$ in $C([\varepsilon, T])$ is dense, $\left\langle\tilde{\mu}, \mathcal{G}^{\prime}(u) v\right\rangle=0$ for all $v \in L^{2}\left(Q_{\omega}\right)$ implies $\tilde{\mu}=0$.

We next prove that when state constraint is active over an interval, the Cantor part of the measure is also null.

Lemma 14. Let $(u, y)$ be a regular extremal of $(P)$. Assume that the state constraint is active over an interval $\left[t_{1}, t_{2}\right]$, where $0 \leq t_{1}<t_{2} \leq T$. Then $\mu$ is absolutely continuous over $\left[t_{1}, t_{2}\right]$.

Proof. It suffices to prove that the expression in the r.h.s. of (69) belongs to $W^{1,1}\left(t_{1}, t_{2}\right)$. Since by assumption the denominator is far from zero and belongs to $W^{1,1}\left(t_{1}, t_{2}\right)$, it suffices to check it for each term of the numerator. This is obviously true for the last two terms, and for the first one we observe that for $t \in\left(t_{1}, t_{2}\right)$ :

$$
|\nabla y(t)|^{2}=\left|\nabla y\left(t_{1}\right)\right|^{2}-\int_{t_{1}}^{t} \int_{\Omega} \Delta y(x, s) y_{t}(x, s) \mathrm{d} x \mathrm{~d} t
$$

Indeed this holds when $y$ is smooth enough, and since each side of the equality is a continuous function in $H^{2,1}(Q)$, and the latter is the closure (for its norm) of the set of $C^{\infty}$ functions over $Q,(78)$ holds. The conclusion follows.

\subsection{Arc based alternative formulation}

We next present a variant of the alternative formulation that is useful when formulating shooting algorithms; see Bonnans and Hermant [15] for related results in a finite dimensional setting. Let us start with some definitions. A boundary arc (resp. interior arc) is a maximal interval of positive measure $\mathcal{I}$ such that $g(y)(t)=0$ (resp. $g(y)(t)<0$ ), for all $t \in \mathcal{I}$. Left and right endpoints of a boundary arc $\left[\tau_{e n}, \tau_{e x}\right]$ are called entry and exit point, respectively. A touch point $\tau_{t o}$ is an isolated contact point, satisfying $g(y)\left(\tau_{t o}\right)=0$ and $g(y)(t)<0$ for $t \neq \tau_{t o}$ in the neighborhood of $\tau_{t o}$. The endpoints of interior arcs belonging to $(0, T)$ are called junction points (or times). If the set of junction points of a trajectory is finite, then it is of the form

$$
\mathcal{T}=\mathcal{T}_{\text {en }} \cup \mathcal{T}_{\text {ex }} \cup \mathcal{T}_{\text {to }}
$$

with $\mathcal{T}_{\text {en }}, \mathcal{T}_{\text {ex }}, \mathcal{T}_{\text {to }}$ the disjoint (and possible empty) subsets of respectively entry, exit and touch points. We denote by $I_{b}$ the union of boundary arcs, i.e. $I_{b}=$ $\bigcup_{i=1}^{N_{b}}\left[\tau_{i}^{e n}, \tau_{i}^{e x}\right]$ for $\mathcal{T}_{\text {en }}:=\left\{\tau_{1}^{e n}<\cdots<\tau_{N_{b}}^{e n}\right\}$ and similar definition of $\mathcal{T}_{\text {ex }}$, and we have $I(g(y))=\mathcal{T}_{\text {to }} \cup I_{b}$.

Definition 15. We say that a trajectory $(u, y)$ is solution of the alternative formulation, if there exist $p_{1} \in L^{2}(Q), \mu_{1} \in B V(0, T)$ and alternative jump 
parameters $\nu_{\mathcal{T}_{\text {en }}}^{1}$ such that, setting $Q_{-\mathcal{T}}:=\Omega \times([0, T] \backslash \mathcal{T})$ :

$$
\begin{aligned}
y_{t}-\Delta y+\gamma y^{3} & =i_{\omega} \text { u a.e. }(x, t) \in Q \\
y(0, \cdot)=y_{0}, & \\
y=0 \text { on } \Sigma & \\
-p_{1, t}-\Delta p_{1}+3 \gamma y^{2} p_{1} & =y-y_{d}+\left(2 \Delta y-6 \gamma y^{3}+i_{\omega} u\right) \mu_{1}(t) \text { on } Q_{-\mathcal{T}}(83) \\
p_{1}(\cdot, T) & =0 \\
p_{1}(\cdot, t)_{\mid \Sigma} & =0 \\
N u+\chi_{\omega}^{*}\left(p_{1}+\mu_{1} y\right) & =0 \text { a.e. on }[0, T] \times \Omega, \\
g(y)\left(\tau_{\text {en }}\right) & =0, \tau_{\text {en }} \in \mathcal{T}_{\text {en }}, \frac{\mathrm{d}}{\mathrm{d} t} g(u, y)=0 \text { on } I_{b} \\
\mu_{1}(t) & =0 \text { on }[0, T] \backslash I_{b} \\
{\left[p_{1}(\cdot, \tau)\right] } & =-\nu_{\tau}^{1} y(\tau) \text { for all } \tau \in \mathcal{T}_{\text {en }} . \\
{\left[p_{1}(\cdot, \tau)\right] } & =0 \text { for all } \tau \in \mathcal{T}_{\text {ex }} .
\end{aligned}
$$

A solution of the alternative formulation is said to satisfy the additional conditions, if the conditions below hold:

$$
\begin{array}{r}
g(y)(t)<0 \text { a.e. on }[0, T] \backslash I_{b} \\
\mu_{1} \text { is nonincreasing on } \operatorname{int}\left(I_{b}\right) \\
\nu_{\tau_{e n}}^{1}=\mu_{1}\left(\tau_{e n}^{+}\right), \tau_{e n} \in \mathcal{T}_{e n}, \mu_{1}\left(\tau_{e x}^{-}\right)=0, \tau_{e x} \in \mathcal{T}_{\text {ex }} .
\end{array}
$$

Consider the following relations:

$$
\begin{aligned}
\nu_{\tau_{e n}} & =-\mu\left(\tau_{e x}\right)-\mu\left(\tau_{e n}\right)=\mu\left(\left[\tau_{e n}, \tau_{e x}\right]\right), \\
\mu_{1}(t) & =-\mu(t)-\Sigma_{\tau \in \mathcal{T}_{e n}} \nu_{\tau}^{1} \mathbf{1}_{[0, \tau)}(t) \\
p_{1} & =p^{1}+\Sigma_{\tau \in \mathcal{T}_{e n}} \nu_{\tau}^{1} \mathbf{1}_{[0, \tau)}(t) y(t)
\end{aligned}
$$

We have the following propositions.

Proposition 16. Let $(u, y) \in F(P)$ satisfy the qualification condition. Then $(u, y)$ is a extremal iff it satisfies both the alternative formulation (80)-(90) and the additional conditions (91)-(93), the relations between the adjoint states and multiplier being given by (94)- (96).

For the proof it suffices to substitute in the alternative formulation the expressions of $\left(p_{1}, \mu_{1}\right)$ in (94)-(96). Details are left to the reader. The derivation is analogous to the one in e.g. [16. The above formulation opens the way to the resolution of the optimality system by shooting methods. This extension is not obvious, however, in view of the ill-posedness of the backward heat equation.

\section{Second-order optimality condition}

\subsection{Second-order necessary optimality condition}

Since the seminal work by Kawasaki 26, 27, 28, we know that second-order necessary optimality conditions involve the difference of th Hessian of Lagragian with a "sigma-term" taking into account the curvature of the convex set involved 
in the constraint statement (in the context of our state constrained optimal control problems, the cone $C([0, T])_{\text {- of nonpositive continuous functions over }}$ $[0, T])$. This was set in a general setting by Cominetti 22, and examples of computations of the sigma-term for various function spaces, including those of continuous functions over a compact set, are provided in Cominetti and Penot 22.

The main result of Bonnans and Hermant [17] is that, under weak conditions, for the optimal control of o.d.e.'s, this sigma-term reduces to the contribution of isolated contact points (called touch points), that are equal to the product of the jump of the multiplier times a quadratic form of the critical direction at the touch point. In addition, for first-order state constraints, the contribution of "regular" touch points is also zero. We will obtain a similar result in our context. In fact we improve here the method of [17] by avoiding any hypothesis on the second-derivative of the state constraint at junction points.

Th Lagrangian of problem $(P)$ (in the formalism where both the state and control variables appear) in qualified form is the function $L: L^{2}\left(Q_{\omega}\right) \times H_{\Sigma}^{2,1}(Q) \times$ $L^{2}(Q) \times H_{0}^{1}(\Omega) \times M([0, T])$ defined by

$$
\begin{aligned}
L(u, y, p, q, \mu):= & J(u, y)+\int_{Q} p\left(\Delta y-\gamma y^{3}+i_{\omega} u-y_{t}\right) \mathrm{d} x \mathrm{~d} t \\
& +\int_{0}^{T} g(y(t)) \mathrm{d} \mu(t)+\int_{\Omega} q(x)\left(y(x, 0)-y_{0}(x)\right) \mathrm{d} x .
\end{aligned}
$$

Its second-order directional derivative in direction $(v, z)$ is

$$
\Delta(v, z):=N\|v\|_{2}^{2}+\int_{Q}(1-6 \gamma p(x, t) y(x, t)) z(x, t)^{2} \mathrm{~d} x \mathrm{~d} t+\int_{0}^{T}|z(t)|_{2}^{2} d \mu(t) .
$$

We say that $(v, z) \in L^{2}(Q) \times H_{\Sigma}^{2,1}(Q)$ is a critical direction if (i) it satisfies the linearized state equation (23) and (ii) it is tangent to the state constraints and satisfies the complementarity condition with the multiplier $\mu$, i.e.,

$$
\begin{array}{lll}
g^{\prime}(y(t)) z(t) & \leq 0 & \text { over } I(g(y)) \\
g^{\prime}(y(t)) z(t) & =0 &
\end{array}
$$

Denote by $C(u, y)$ the set of critical directions associated with $(u, y) \in F(P)$. We say that the contact set has a finite structure if it is a finite union of touch points and boundary arcs. In that case, we will say that the hypothesis of strict complementarity holds if the support of the measure $\mathrm{d} \mu$ is the union of the boundary arcs. In that case, a direction $(v, z)$ satisfying (23) is critical iff it satisfies

$$
\left\{\begin{array}{lll}
g^{\prime}(y(t)) z(t) & =0 & \text { over boundary arcs, } \\
\left.g^{\prime}(y(\tau)) z(\tau)\right) & \leq 0 & \text { for each touch point } \tau
\end{array}\right.
$$

Theorem 17. Let $(u, y)$ be a qualified local solution of $(P)$, with associated multiplier $\mu$ and costate $p$. If the contact set has a finite structure and the hypothesis of strict complementarity holds, then

$$
\Delta(v, z) \geq 0, \quad \text { for all }(v, z) \in C(u, y) .
$$

The proof will use the following corollary of lemma 8 
Corollary 18. Let $(\bar{v}, \bar{z})$ be solution of the linearized equation. Consider the linearized equation with r.h.s. in feedback form:

$$
v=\bar{v}+(\eta(z, t)-\eta(\bar{z}, t)+\nu(t) \kappa(t)) \chi_{\omega} y .
$$

When $\nu \rightarrow 0$ in $L^{2}(0, T)$, we have that $v \rightarrow \bar{v}$ in $L^{2}\left(Q_{\omega}\right)$, and in addition

$$
\frac{\mathrm{d}}{\mathrm{d} t}\left(g^{\prime}(y) z\right)=\nu_{1}(t):=\nu(t)+\frac{\mathrm{d}}{\mathrm{d} t}\left(g^{\prime}(y) \bar{z}\right) \quad \text { over } \mathcal{T} .
$$

Proof. By lemma 8 where here $f=\bar{v}+\left(\nu_{1} \kappa(t)-\eta_{\bar{z}}\right) \chi_{\omega} y$, the equation is wellposed. Since

$$
\frac{\mathrm{d}}{\mathrm{d} t}\left(g^{\prime}(y) \bar{z}\right)=\int_{\omega} \bar{v} y \mathrm{~d} x-\eta(\bar{z}, t)
$$

relation (104) follows from (46). In addition, the mapping $\nu \mapsto(v, z)$ is continuous $L^{2}(0, T) \rightarrow L^{2}\left(Q_{\omega}\right) \times H_{\Sigma}^{2,1}(Q)$ (as being a composition of continuous mappings), and $(v, z)=(\bar{v}, \bar{z})$ when $\nu=0$. The conclusion follows.

Proof of theorem 17. We apply the extended polyhedricity theory of 18, Section $3.2 .3])$. Denote by $C_{R}(u, y)$ the set of radial critical direction, i.e., of critical directions $(v, z)$ such that $g(y)+\sigma g^{\prime}(y) z \in K$ for some $\sigma>0$. It is known that $\Delta(v, z) \geq 0$, for all $(v, z) \in C_{R}(u, y)$, and since $\Delta(v, z)$ is a continuous function, this still holds over the closure $\bar{C}_{R}(u, y)$. We will next check the extended polyhedricity assumption $\bar{C}_{R}(u, y)=C(u, y)$ under which, by the above arguments, the conclusion holds.

Let us check this hypothesis by induction on $N_{t o t}:=N_{t c}+N_{b}$, where $N_{t c}$ and $N_{b}$ are the number of touch points and boundary arcs, respectively. The result obviously holds when $N_{t o t}=0$; assuming that it holds for $N_{t o t}-1$, let $t_{0} \in(0, T)$ be such that $g\left(y\left(t_{0}\right)\right)<0$ and that $I(g(y)) \cap\left(t_{0}, T\right]$ is the last connected component of $I(g(y))$.

Give a critical direction $(v, z)$, consider the restriction of $(y, u, z, v)$ to $\left[0, t_{0}\right]$. Then $(v, z)$ is critical over $\left[0, t_{0}\right]$ in the sense that it satisfies the linearized state constraint over the active set while being complementary to the multiplier. Since $I(g(y))$ has $N_{t o t}-1$ connected components on $\left[0, t_{0}\right]$, by our induction argument, for any $\varepsilon>0$, there exists a "radial critical direction over $\left(0, t_{0}\right)$ ", i.e., $v_{\varepsilon} \in L^{2}\left(\omega \times\left(0, t_{0}\right)\right)$ and $z_{\varepsilon} \in H_{\Sigma}^{2,1}\left(\Omega \times\left(0, t_{0}\right)\right)$ such that, for some $\sigma_{\varepsilon}>0$, $g(y)+\sigma_{\varepsilon} g^{\prime}(y) z_{\varepsilon} \leq 0$ over $\left[0, t_{0}\right],(v, z)$ satisfy the linearized state equation over $\left(0, t_{0}\right)$, and $\left\|v_{\varepsilon}-v\right\|_{2}+\left\|z_{\varepsilon}-z\right\|_{2,1} \leq \varepsilon$. Since the trace at time $t_{0}$ is a continuous mapping $H_{\Sigma}^{2,1}\left(\Omega \times\left(0, t_{0}\right)\right) \rightarrow H_{0}^{1}(\Omega)$, there exists $c_{1}>0$ such that $\left\|z_{\varepsilon}\left(\cdot, t_{0}\right)-z\left(\cdot, t_{0}\right)\right\|_{H_{0}^{1}(\Omega)} \leq c_{1} \varepsilon$. Therefore, for some $c_{2}>0$ not depending on $(v, z)$, we have that

$$
\left|\left(g^{\prime}(y)\left(\bar{z}-z_{\varepsilon}\right)\right)\left(t_{0}\right)\right| \leq c_{2} \varepsilon .
$$

Let $\tau$ be the smallest point of the last connected component of $I(g(y))$; we can increase $t_{0}$ if necessary in order to be sure that $\kappa(t)>0$ over $\left[t_{0}, \tau\right]$. Integrating (104) over $\left(t_{0}, \tau\right)$, obtain

$$
\left(g^{\prime}(y) z_{\varepsilon}\right)(\tau)=\left(g^{\prime}(y) z_{\varepsilon}\right)\left(t_{0}\right)+\int_{t_{0}}^{\tau} \nu(t) \mathrm{d} t+\left[g^{\prime}(y) \bar{z}\right]_{t_{0}}^{\tau} .
$$

Using (106), get

$$
\left|\left(g^{\prime}(y)\left(z_{\varepsilon}-\bar{z}\right)\right)(\tau)-\int_{t_{0}}^{\tau} \nu(t) \mathrm{d} t\right| \leq c_{2} \varepsilon .
$$


We next have to distinguish two cases.

(i) The last connected subset of $I(g(y))$ is the touch point $\tau$. Take

$$
\nu(t):=\left\{\begin{array}{cl}
-2 \varepsilon\left(c_{2}+1\right) /\left(\tau-t_{0}\right) & \text { when } t<\tau, \\
0 & \text { when } t \in[\tau, T] .
\end{array}\right.
$$

Since $\left(g^{\prime}(y) \bar{z}\right)(\tau)=0$, we have then $\left(g^{\prime}(y) z_{\varepsilon}\right)(\tau) \leq-\varepsilon$, so that for small $\sigma>0$, $g(y)+g^{\prime}(y) z_{\varepsilon} \leq 0$ over $\left[t_{0}, T\right]$, and at the same time we have constructed $v_{\varepsilon}$ such that $\left\|v_{\varepsilon}-\bar{v}\right\|_{2}+\left\|z_{\varepsilon}-\bar{z}\right\|_{2,1}=O(\varepsilon)$. So we have constructed a radial direction $\left(v_{\varepsilon}, z_{\varepsilon}\right)$ converging to $(v, z)$.

(ii) The last connected subset of $I(g(y))$ is the boundary arc $\left[\tau, \tau^{\prime}\right]$. As a first step, take $\nu(t)$ equal to some constant $c_{3}$ so that $\left(g^{\prime}(y) z\right)(\tau-\varepsilon)=0$. Using the relation similar to (107) but at time $\tau-\varepsilon$, get

$$
0=\left(g^{\prime}(y) z_{\varepsilon}\right)(\tau-\varepsilon)=c_{3}\left(\tau-\varepsilon-t_{0}\right)+\left(g^{\prime}(y)\left(z_{\varepsilon}-\bar{z}\right)\right)\left(t_{0}\right)+\left(g^{\prime}(y) \bar{z}\right)(\tau-\varepsilon) .
$$

Since $g^{\prime}(y) \bar{z}$ is a continuous fonction, we have that $\left|\left(g^{\prime}(y) z\right)(\tau-\varepsilon)\right|=o(1)$, and hence for $\varepsilon \leq \frac{1}{2}\left(\tau-t_{0}\right)$, it suffices to take $c_{3}=o(1)$.

The second step is to set $\left(g^{\prime}(y) z\right)(t)=0$, for all $t \in[\tau-\varepsilon, \tau]$, i.e., $\nu(t)=$ $-\frac{\mathrm{d}}{\mathrm{d} t}\left(g^{\prime}(y) \bar{z}\right)(t)$, and hence, $\|\nu\|_{L^{2}(\tau-\varepsilon, \tau)}=o(1)$. On $\left[\tau, \tau^{\prime}\right]$ we take of course $\nu=0$, and if $\tau^{\prime}<T$, we set $\nu$ so that $\left(g^{\prime}(y) z\right)(t)=0$, for all $t \in\left[\tau^{\prime}, \tau^{\prime}+\varepsilon\right]$, which similarly implies $\|\nu\|_{L^{2}\left(\tau^{\prime}, \tau+\varepsilon\right)}=o(1)$. So again we have constructed a radial critical direction converging to $(v, z)$.

\subsection{Second-order sufficient optimality condition}

We obtain in this section no-gap second-order optimality conditions.

Theorem 19. Let $(u, y)$ be a regular extremal of $(P)$. Then a sufficient condition for the quadratic growth condition (20) is

$$
\Delta(v, z)>0, \quad \text { for all }(v, z) \in C(u, y) \backslash\{0\} .
$$

If, in addition, the contact set has a finite structure, then (111) is a necessary condition for quadratic growth.

Proof. a) The sufficiency condition follows from [18, Section 3.3]; for the sake of completeness, we give a direct proof. Assume that (111) holds, and that a feasible sequence $\left(u_{k}, y_{k}\right)$ for $(P)$ satisfies $\left(u_{k}, y_{k}\right) \neq(u, y)$ for all $k,\left(u_{k}, y_{k}\right) \rightarrow$ $(u, y)$, and

$$
\mathcal{J}\left(u_{k}\right)=J\left(u_{k}, y_{k}\right) \leq J(u, y)+o\left(\left\|u_{k}-u\right\|_{2}^{2}\right) .
$$

Set $\beta_{k}:=\left\|u_{k}-u\right\|_{2}$ and $v_{k}:=\left(u_{k}-u\right) / \beta_{k}$. Then $u_{k}=u+\beta_{k} v_{k}$. Since $\left\|v_{k}\right\|_{2}=1$, extracting if necessary a subsequence, we may assume the existence of $v \in L^{2}(\Omega)$ such that $v_{k} \rightarrow v$ (weak convergence). Since $\mathcal{J}\left(u_{k}\right)=\mathcal{J}(u)+\beta_{k} \mathcal{J}^{\prime}(u) v_{k}+o\left(\beta_{k}\right)$, we deduce from (112) that $\mathcal{J}^{\prime}(u) v \leq 0$. Similarly, since

$$
T_{K}(\mathcal{G}(u)) \supset \frac{K-\mathcal{G}\left(u_{k}\right)}{\beta_{k}}=\mathcal{G}^{\prime}(u) v_{k}+o(1),
$$

and since $T_{K}(\mathcal{G}(u))$ is a (weakly) closed convex set, we obtain that $\mathcal{G}^{\prime}(u) v \in$ $T_{K}(\mathcal{G}(u))$. It follows that $v$ is a critical direction. Using $L\left(u_{k}, y_{k}, p, q, \mu\right) \leq$ $J\left(u_{k}, y_{k}\right)$ and $L(u, y, p, q, \mu)=J(u, y)$, deduce from (112) that

$$
0 \geq L\left(u_{k}, y_{k}, p, q, \mu\right)-L(u, y, p, q, \mu)+o\left(\beta_{k}^{2}\right)=\frac{1}{2} \beta_{k}^{2} \Delta\left(v_{k}, z_{k}\right)+o\left(\beta_{k}^{2}\right) .
$$


Since $\Delta(\cdot, \cdot)$ is w.l.s.c., obtain $\Delta(v, z) \leq 0$, which by (111), since $(v, z)$ is a critical direction, implies $(v, z)=0$, and hence, $\Delta\left(v_{k}, z_{k}\right) \rightarrow \Delta(v, z)$. Since the mapping $v \rightarrow z$ (solution of the linearized state equation) is compact $L^{2}\left(Q_{\omega}\right) \rightarrow L^{2}(Q)$, it follows that $\left\|v_{k}\right\|_{2} \rightarrow\|v\|_{2}$, and therefore $v_{k} \rightarrow v$ (for the strong topology), contradicting $\left\|v_{k}\right\|_{2}=1$.

b) Let $(u, y) \in F(P)$ be qualified, have a finite structure, and satisfy the quadratic growth condition. Then for $\varepsilon>0$ small enough $(u, y)$ is a local solution of the same problem with $N$ changed into $N-\frac{1}{2} \varepsilon$. Since this does not change the expression of the first-order optimality conditions at the point $(u, y)$, the costate $p$ and multiplier $\mu$ are the same, while the perturbed Hessian of Lagrangian is $\Delta_{\varepsilon}(v, z)=\Delta(v, z)-\varepsilon\|v\|_{2}^{2}$. Since $\Delta_{\varepsilon}(v, z) \geq 0$ by theorem [19] the conclusion follows.

\section{Sensitivity analysis}

We consider now a family of optimal control problems parameterized by an additional $f \in L^{2}(Q)$ on the r.h.s. of the state equation. The perturbed state equation is therefore

$$
\begin{aligned}
y_{t}-\Delta y+\gamma y^{3} & =f+i_{\omega} u \text { in } Q \\
y & =0 \text { over } \Sigma \\
y(\cdot, 0) & =y_{0} \text { over } \Omega .
\end{aligned}
$$

Let $(\bar{u}, \bar{y})$ be a local solution of $(P)$ satisfying the quadratic growth condition (20) for some $\theta>0$ and $\rho>0$. Assume that they satisfy the qualification condition, and let $(\bar{p}, \bar{\mu})$ denote the associated costate and Lagrange multiplier. Consider the localizing constraint

$$
\|u-\bar{u}\|_{2} \leq \rho
$$

The perturbed optimal control problem is

$$
\operatorname{Min}_{(u, y) \in L^{2}\left(Q_{\omega}\right) \times H^{2,1}(Q)} J(u, y) \text { subject to (115)-(117) } \text { and (118). }
$$

Denote by $v(f)$ the value of problem $\left(P_{f}\right)$. Using the methodology of [13, 18, we are able to perform a sensitivity analysis along a path of the form

$$
f(\sigma):=\sigma f_{1}+\frac{1}{2} \sigma^{2} f_{2}+o\left(\sigma^{2}\right)
$$

where $f_{1}$ and $f_{2}$ are given in $L^{2}(Q)$. Consider the following perturbed linearized equation

$$
z_{t}-\Delta z+3 \gamma \bar{y}^{2} z=f_{1}+i_{\omega} v \text { in } Q ; \quad z=0 \text { on } \Sigma, \quad z(\cdot, 0)=0 .
$$

The related linearized optimization problem is

$\underset{(v, z) \in L^{2}\left(Q_{\omega}\right) \times H^{2,1}(Q)}{\operatorname{Min}} J^{\prime}(\bar{u}, \bar{y})(v, z) ; \quad g^{\prime}(\bar{y}(t)) z(t) \leq 0 \quad$ over $I(g(\bar{y}))$; (120) holds.

Note that $S\left(L_{f_{1}}\right)$ coincides with the critical cone if $f_{1}=0$. We start with a technical lemma concerning the value of the linearized problem $\left(L_{f_{1}}\right)$. 
Lemma 20. We have that $\operatorname{val}\left(L_{f_{1}}\right)=\int_{Q} \bar{p}(x, t) f_{1}(x, t) \mathrm{d} x \mathrm{~d} t$. In addition, the set $S\left(L_{f_{1}}\right)$ is non empty.

Proof. Since $(\bar{u}, \bar{y})$ satisfies the qualification condition, and in view of the uniqueness of the Lagrange multiplier, we deduce from [13. Prop. 3.1] that (i) $\left(L_{f_{1}}\right)$ has the same Lagrange multiplier $\bar{\mu}$ as the original problem $(P)$, (ii) $(v, z) \in S\left(L_{f_{1}}\right)$ iff $(v, z) \in F\left(L_{f_{1}}\right)$ with strict complementarity with $\bar{\mu}$, i.e.

$$
g^{\prime}(\bar{y}(t)) z(t)=0 \text { over } \operatorname{supp}(\bar{\mu})
$$

and (iii) that the formula for $\operatorname{val}\left(L_{f_{1}}\right)$ holds. The nonemptiness of the solution set follows from the fact that, in view of Lemma 8 we can control the time derivative of the state constraint over a neigbourhood of the contact set, and therefore it is possible to set the first-order variation of the state constraint to zero over the contact set.

Next we need an estimate of stability of approximate solutions. The Lagrangian of the family of perturbed problems (by abuse of notation, denoted in the same way) is

$$
\begin{aligned}
L(u, y, f, p, q, \mu):= & J(u, y)+\int_{Q} p\left(\Delta y-\gamma y^{3}+f+i_{\omega} u-y_{t}\right) \mathrm{d} x \mathrm{~d} t \\
& +\int_{0}^{T} g(y(t)) \mathrm{d} \mu(t)+\int_{\Omega} q(x)\left(y(x, 0)-y_{0}\right) \mathrm{d} x .
\end{aligned}
$$

It is easily checked that the value of the multiplier for the initial condition is $\bar{q}=-\bar{p}(0)$.

Lemma 21. Let $(\bar{u}, \bar{y})$ be a qualified local solution of $(P)$ satisfying the quadratic growth condition (20). If $\rho$ is small enough, then (i) when $\|f\|_{2}$ is small enough, problem $\left(P_{f}\right)$ has a nonempty (necessarily bounded) set of solutions, (ii) if $f_{k} \rightarrow$ 0 in $L^{2}(Q)$, and $u_{k}$ is a sequence of $o\left(\left\|f_{k}\right\|_{2}^{2}\right)$ solutions of problem $\left(P_{f_{k}}\right)$, we have that $\left\|u_{k}-\bar{u}\right\|_{2}=O\left(\left\|f_{k}\right\|_{2}\right)$.

Proof. Since the Implicit Function Theorem may be applied to the study of the state equation (115)-(117), denoting its solution by $y_{f, u}$, we have that, in the vicinity of $(f=0, \bar{u})$, the mapping $(f, u) \rightarrow y_{f, u}$ is well-defined and of class $C^{\infty}$. In particular, when $\rho$ and $\|f\|_{2}$ are small enough, $y_{f, u}$ is uniformly bounded in $H_{\Sigma}^{2,1}(Q)$. Therefore we can pass to the limit in a minimizing sequence of problem $\left(P_{f}\right)$, using standard compactness arguments in order to deal with the nonlinear term of the state equation. Point (i) follows.

(ii) If the conclusion does not hold, for arbitrarily small $\rho>0$ there exist sequences $f_{k} \rightarrow 0$ in $L^{2}(Q)$ and $u_{k}$ of $o\left(\sigma^{2}\right)$ solutions of problem $\left(P_{f_{k}}\right)$ such that $\left\|f_{k}\right\|_{2}=o\left(\left\|u_{k}-\bar{u}\right\|_{2}\right)$. We may write $\sigma_{k}:=\left\|u_{k}-\bar{u}\right\|_{2}$, and $u_{k}=\bar{u}+\sigma_{k} v_{k}$, with $\left\|v_{k}\right\|_{2}=1$. Extracting if necessary a subsequence, assume that $v_{k} \rightarrow \bar{v}$. From a first-order expansion of the cost function and constraints we easily obtain that $\bar{v}$ is a critical direction. Now

$$
L\left(u_{k}, y_{k}, f_{k}, \bar{p}, \bar{q}, \bar{\mu}\right) \leq J\left(u_{k}, y_{k}\right) \leq \operatorname{val}\left(P_{f_{k}}\right)+o\left(\left\|f_{k}\right\|_{2}^{2}\right) .
$$

From a second-order expansion of the Lagrangian, whose partial derivative w.r.t. $(u, y)$ is zero at $(\bar{u}, \bar{y}, f=0)$, and since $L(\bar{u}, \bar{y}, 0, \bar{p}, \bar{q}, \bar{\mu})=J(\bar{u}, \bar{y})$, denoting 
by $z_{k}$ the solution of (120) with $v:=v_{k}$, get $\Delta\left(v_{k}, z_{k}\right) \leq o(1)$, and hence, $\Delta(\bar{v}, \bar{z}) \leq \liminf _{k} \Delta\left(v_{k}, z_{k}\right) \leq 0$. Since $(\bar{v}, \bar{z})$ is a critical direction, it follows that $(\bar{v}, \bar{z})=0$, and hence $\Delta\left(v_{k}, z_{k}\right) \rightarrow \Delta(\bar{v}, \bar{z})$, which implies $v_{k} \rightarrow \bar{v}$. This cannot be since $v_{k}$ is of unit norm and $\bar{v}=0$.

Consider the quadratic subproblem

$$
\operatorname{Min}_{(v, z) \in S\left(L_{f_{1}}\right)} \Delta(v, z)+\int_{Q} \bar{p}(x, t) f_{2}(x, t) \mathrm{d} x \mathrm{~d} t
$$

Theorem 22. Let $(\bar{u}, \bar{y})$ be a qualified local solution of $(P)$ satisfying the quadratic growth condition (20). Then a) we have the following expansion

$$
v(f(\sigma))=\operatorname{val}(P)+\sigma \operatorname{val}\left(L_{f_{1}}\right)+\frac{1}{2} \sigma^{2} \operatorname{val}(Q)+o(\sigma) .
$$

b) In addition we have that if $\left(u_{\sigma}, y_{\sigma}\right)$ is a path of $o\left(\sigma^{2}\right)$ solutions, then $\| u_{\sigma}-$ $\bar{u} \|_{2}=O(\sigma)$, each weak limit-point in $L^{2}\left(Q_{\omega}\right)$ is a strong limit-point, and is solution of problem $(Q)$. If the latter has a unique solution $\bar{v}$, then a path $u_{\sigma}$ of $o\left(\sigma^{2}\right)$ solutions of $\left(P_{f(\sigma)}\right)$ satisfies

$$
u_{\sigma}=\bar{u}+\sigma \bar{v}+o(\sigma)
$$

Proof. a) Apply [18 Thms 4.94]. Its hypothesis are (i) directional regularity, that follows from the qualification condition, (ii) existence of a $o\left(\sigma^{2}\right)$ solution path for problem $\left(P_{f(\sigma)}\right)$, which holds in view of lemma 21] (iii) the directional extended polyhedricity condition which is consequence of extended polyhedricity. The conclusion is, with numbering of equations of [18, that the expansion (4.235), based on the expressions defined in (4.222)-(4.224), holds. Therefore, since (as easily checked) val $(Q)$ is finite, (our equation) (124) holds.

b) This is an obvious consequence of [18, Thm 4.95].

\section{References}

[1] F. Abergel and R. Temam. Optimality conditions for some nonqualified problems of distributed control. SIAM J. Control Optim., 27(1):1-12, 1989.

[2] R.A. Adams. Sobolev spaces. Academic Press, New York, 1975.

[3] N. Arada and J.-P. Raymond. Optimal control problems with mixed control-state constraints. SIAM J. Control Optim., 39(5):1391-1407 (electronic), 2000.

[4] N. Arada and J.-P. Raymond. Dirichlet boundary control of semilinear parabolic equations. II. Problems with pointwise state constraints. Appl. Math. Optim., 45(2):145-167, 2002.

[5] Nadir Arada and J.-P. Raymond. Minimax control of parabolic systems with state constraints. SIAM J. Control Optim., 38(1):254-271 (electronic), 1999.

[6] Nadir Arada and J.-P. Raymond. Necessary optimality conditions for control problems and the Stone-Čech compactification. SIAM J. Control Optim., 37(4):1011-1032 (electronic), 1999. 
[7] J. W. Bebernes and D. R. Kassoy. A mathematical analysis of blowup for thermal reactions - the spatially nonhomogeneous case. SIAM J. Appl. Math., 40(3):476-484, 1981.

[8] J. F. Bonnans and E. Casas. On the choice of the function spaces for some state-constrained control problems. Numer. Funct. Anal. Optim., 7(4):333$348,1984 / 85$.

[9] J.F. Bonnans. Contrôle optimal de systèmes instables gouvernés par des équations aux dérivées partielles non linéaires. $\mathrm{PhD}$ thesis, Université Paris IX Dauphine, 1982.

[10] J.F. Bonnans. Analysis and control of a nonlinear parabolic unstable system. Large Scale Systems, 6:249-262, 1984.

[11] J.F. Bonnans and E. Casas. Contrôle de systèmes elliptiques semilinéaires comportant des contraintes sur l'état. In H. Brézis and J.L. Lions, editors, Nonlinear partial differential equations and their applications, Collège de France seminar vol. VII, volume 166 of Pitman Research Notes in Mathematics, pages 69-86. Longman Scientific \& Technical, New York, 1988.

[12] J.F. Bonnans and E. Casas. Optimal control of semilinear multistate systems with state constraints. SIAM Journal on Control and Optimization, $27: 446-455,1989$.

[13] J.F. Bonnans and R. Cominetti. Perturbed optimization in Banach spaces I: A general theory based on a weak directional constraint qualification, II: A theory based on a strong directional qualification, III: Semi-infinite optimization. SIAM Journal on Control Optimization, 34:1151-1171, 11721189 and 1555-1567, 1996.

[14] J.F. Bonnans and A. Hermant. Second-order analysis for optimal control problems with pure state constraints and mixed control-state constraints. Annals of I.H.P. - Nonlinear Analysis. DOI: 10.1016/j.anihpc.2007.12.002.

[15] J.F. Bonnans and A. Hermant. Well-posedness of the shooting algorithm for state constrained optimal control problems with a single constraint and control. SIAM J. Control Optimization, 46(4):1398-1430, 2007.

[16] J.F. Bonnans and A. Hermant. Stability and sensitivity analysis for optimal control problems with a first-order state constraint. ESAIM:COCV, 14(4):825-863, 2008. Available at http://hal.inria.fr/inria-00087573.

[17] J.F. Bonnans and A. Hermant. No gap second order optimality conditions for optimal control problems with a single state constraint and control. Mathematical Programming, Series B, 117:21-50, 2009. Online First DOI 10.1007/s10107-007-0167-8.

[18] J.F. Bonnans and A. Shapiro. Perturbation analysis of optimization problems. Springer-Verlag, New York, 2000.

[19] E. Casas, J.-P. Raymond, and H. Zidani. Pontryagin's principle for local solutions of control problems with mixed control-state constraints. SIAM J. Control Optim., 39(4):1182-1203 (electronic), 2000. 
[20] Eduardo Casas. Pontryagin's principle for state-constrained boundary control problems of semilinear parabolic equations. SIAM J. Control Optim., 35(4):1297-1327, 1997.

[21] Eduardo Casas, Juan Carlos de los Reyes, and Fredi Tröltzsch. Sufficient second-order optimality conditions for semilinear control problems with pointwise state constraints. SIAM J. Optim., 19(2):616-643, 2008.

[22] R. Cominetti and J.P. Penot. Tangent sets to unilateral convex sets. Comptes Rendus de l'Académie des Sciences de Paris, Série I, 321:16311636, 1995.

[23] N. Dunford and J. Schwartz. Linear operators, Vol I and II. Interscience, New York, 1958, 1963.

[24] W.W. Hager. Lipschitz continuity for constrained processes. SIAM J. Control Optimization, 17:321-338, 1979.

[25] D.H. Jacobson, M.M. Lele, and J.L. Speyer. New necessary conditions of optimality for control problems with state-variable inequality contraints. J. of Mathematical Analysis and Applications, 35:255-284, 1971.

[26] H. Kawasaki. An envelope-like effect of infinitely many inequality constraints on second order necessary conditions for minimization problems. Mathematical Programming, 41:73-96, 1988.

[27] H. Kawasaki. The upper and lower second order directional derivatives of a sup-type function. Mathematical Programming, 41:327-339, 1988.

[28] H. Kawasaki. Second order necessary optimality conditions for minimizing a sup-type function. Math. Programming, 49(2, (Ser. A)):213-229, 1990/91.

[29] J.-L. Lions. Contrôle des systèmes distribués singuliers. Dunod, Paris, 1983.

[30] J.-L. Lions and E. Magenes. Problèmes aux limites non homogènes et applications. Vol. 1. Travaux et Recherches Mathématiques, No. 17. Dunod, Paris, 1968.

[31] H. Maurer. On the minimum principle for optimal control problems with state constraints. Schriftenreihe des Rechenzentrum 41, Universität Münster, 1979.

[32] J. P. Raymond and H. Zidani. Pontryagin's principle for state-constrained control problems governed by parabolic equations with unbounded controls. SIAM J. Control Optim., 36(6):1853-1879 (electronic), 1998.

[33] S.M. Robinson. First order conditions for general nonlinear optimization. SIAM Journal on Applied Mathematics, 30:597-607, 1976.

[34] S.M. Robinson. Stability theorems for systems of inequalities, part II: Differentiable nonlinear systems. SIAM Journal on Numerical Analysis, 13:497-513, 1976. 
[35] Luc Tartar. Topics in nonlinear analysis, volume 13 of Publications Mathématiques d'Orsay 78. Université de Paris-Sud Département de Mathématique, Orsay, 1978.

[36] J. Zowe and S. Kurcyusz. Regularity and stability for the mathematical programming problem in Banach spaces. Journal of Applied Mathematics ES Optimization, 5:49-62, 1979. 


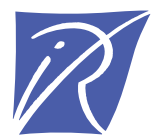

\section{Centre de recherche INRIA Saclay - Île-de-France \\ Parc Orsay Université - ZAC des Vignes \\ 4, rue Jacques Monod - 91893 Orsay Cedex (France)}

Centre de recherche INRIA Bordeaux - Sud Ouest : Domaine Universitaire - 351, cours de la Libération - 33405 Talence Cedex Centre de recherche INRIA Grenoble - Rhône-Alpes : 655, avenue de l'Europe - 38334 Montbonnot Saint-Ismier

Centre de recherche INRIA Lille - Nord Europe : Parc Scientifique de la Haute Borne - 40, avenue Halley - 59650 Villeneuve d'Ascq

Centre de recherche INRIA Nancy - Grand Est : LORIA, Technopôle de Nancy-Brabois - Campus scientifique

615, rue du Jardin Botanique - BP 101 - 54602 Villers-lès-Nancy Cedex

Centre de recherche INRIA Paris - Rocquencourt : Domaine de Voluceau - Rocquencourt - BP 105 - 78153 Le Chesnay Cedex

Centre de recherche INRIA Rennes - Bretagne Atlantique : IRISA, Campus universitaire de Beaulieu - 35042 Rennes Cedex

Centre de recherche INRIA Sophia Antipolis - Méditerranée : 2004, route des Lucioles - BP 93 - 06902 Sophia Antipolis Cedex 